\title{
Thyroid Autoimmunity and Autoimmunity in Chronic Spontaneous Urticaria Linked to Disease Severity, Therapeutic Response, and Time to Remission in Patients with Chronic Spontaneous Urticaria
}

\author{
Kumutnart Chanprapaph (D), Wimolsiri Iamsumang, \\ Penpun Wattanakrai, and Vasanop Vachiramon (i) \\ Division of Dermatology, Department of Internal Medicine, Ramathibodi Hospital, Mahidol University, Bangkok, Thailand \\ Correspondence should be addressed to Kumutnart Chanprapaph; kumutnartp@hotmail.com
}

Received 22 July 2018; Revised 28 September 2018; Accepted 18 October 2018; Published 1 November 2018

Academic Editor: Davinder Parsad

Copyright (c) 2018 Kumutnart Chanprapaph et al. This is an open access article distributed under the Creative Commons Attribution License, which permits unrestricted use, distribution, and reproduction in any medium, provided the original work is properly cited.

Background. Chronic spontaneous urticaria (CSU) is autoimmune in nature and associated with thyroid autoimmunity (TA), but evidence on autoimmunity in relation to CSU progression and prognosis is limited. We evaluated whether TA and autoimmunity in CSU are correlated with disease severity, therapeutic response, and time to remission and establish an association between CSU characteristics linked to thyroid autoantibody. Methods. Medical records of patients diagnosed with urticaria attending outpatient dermatology clinic at a university-based hospital from 2013 to 2017 were retrospectively reviewed. Data on the clinical characteristics, laboratory investigations particularly thyroid antibody titers, autologous serum skin test (ASST) and autologous plasma skin test (APST) results and their link to disease severity, treatments, and time to remission of CSU patients were analyzed. Results. Of 1,096 patients with urticaria, $60.2 \%$ had CSU. Three-hundred patients fulfilled the inclusion criteria for CSU with complete thyroid antibody testing. Positive TA was significantly associated with female gender and age $>35$ years $(\mathrm{p}=0.008)$. Antithyroid peroxidase (anti-TPO)-positive patients suffered from CSU longer than 12 and 18 months compared to anti-TPOnegative patients $(100.0 \%$ vs. $82.6 \%, \mathrm{p}=0.042$, and $100.0 \%$ vs. $75.9 \% \mathrm{p}=0.020$, respectively). The presence of urticarial attacks $>4$ days/week was significantly seen in ASST and APST-positive patients compared to those without (84.6\% vs. $61.3 \%, p=0.011$, and $85.3 \%$ vs. $61.8 \%, p=0.006$, respectively). Positive APST patients were more difficult to treat than those with negative results (61.2\% vs. $37.8 \%, \mathrm{p}=0.017)$. Conclusions. Antithyroid peroxidase is a predictor of time to remission, while autologous skin testing is linked to disease severity (ASST and APST) and therapeutic response (APST) in CSU patients.

\section{Introduction}

Thyroid autoimmunity (TA) is characterized by the production of thyroid autoantibodies and lymphocytic infiltration into the thyroid glands. It is the most common organspecific disorder affecting approximately $5 \%$ of the general population $[1,2]$. Positive thyroid autoantibody is essential for the diagnosis of TA. As the exact pathogenesis is unclear, hereditary and environmental factors appear to be fundamental processes of TA [1].

Chronic spontaneous urticaria (CSU) is defined as the presences of recurrent wheals and flare for a duration of 6 weeks independent of external stimuli [3]. CSU is a common cutaneous disorder with an estimated prevalence of $8-10 \%$ of the general population [4]. CSU has major undesirable effects and significantly impacts the quality of life, mainly due to the high disease activity, sleep deprivation, and psychiatric comorbidity. Therefore, determining factors linking to the severe and resistant cases of CSU is important, as it allows physicians to be more aggressive on their management plans. Majority of cases with CSU have unknown etiology with approximately $30-40 \%$ have autoimmune pathogenesis [5]. Assessing for autoreactivity in-vivo via autologous serum skin test (ASST) and autologous plasma skin test (APST) and in-vitro through basophil histamine release and basophil activation test (BAT) are widely applied. While there is 
evidence to show that BAT with or without the combination of ASST can identify patients with more severe CSU $[6,7]$, there is limited data on whether these results can predict therapeutic response and time to remission in CSU. Coexistence of CSU with major autoimmune diseases has been well documented, particularly autoimmune thyroid diseases (AITD) [8]. The prevalence of positive thyroid autoantibodies in patients with urticaria is significantly higher than nonurticaria controls [1]. Likewise, a recent population-based study has shown that patients with AITD has higher rate of CSU [9].While the association between TA and CSU is well known and is one of the clinical association that contribute to autoimmune hypothesis [6], the relationship between antithyroid antibody and the progression and prognosis of CSU is largely unknown. The objective of this study is to determine the association between TA and autoimmunity of $\mathrm{CSU}$ in relation to CSU disease severity, therapeutic response, and time to remission and establish an association between CSU characteristics linked to thyroid autoantibody.

\section{Material and Methods}

2.1. Study Design. A retrospective study was conducted in a university-based hospital (Ramathibodi Hospital, Mahidol University, Bangkok, Thailand). The medical records of all patients diagnosed with urticaria visiting outpatient dermatologic clinic from January 2013 to May 2017 were retrieved and analyzed. The study was approved from the Mahidol University Institution Review Board (IRB) for human subject research (protocol number 076036). Informed consent was exempted by the board due to the retrospective nature of the study.

2.2. Subjects. Individuals $\geq 15$ years of age who met the diagnostic criteria of CSU, having recurrent wheals and flare of less than 24 hours occurring at least 2 times per week for 6 weeks without identifiable causes, were enrolled in the study. Patients with inducible urticaria (i.e., physical, pressure, cholinergic, cold, drug-induced, and acute urticaria) were excluded. Cases suspected for or had skin biopsy-proven urticarial vasculitis were also excluded from the study. Patients lacking information on both autoimmune thyroid antibodies, including anti-TPO and anti-Tg, were excluded.

2.3. Protocol. Medical record forms were collected for clinical and laboratory information. Data were entered into a database program (Microsoft Excel 2013; Microsoft Corp, Redmond, Washington). Clinical parameters involving patients' gender, age, duration of disease, previous history of AITDs, atopy, systemic symptoms (i.e. angioedema, anaphylaxis), dermographism together with disease severity, therapeutic response, and time to remission were collected. Patients were evaluated for disease severity focusing on duration and frequency of daily attacks, wheal size and number, severity of itch, impairment of work, and disturbance of sleep. Therapeutic response was determined by treatment regimens used, detailed types and dosages of antihistamines, and other medications (H2-receptor antagonist, antileukotrienes, cyclosporin A, omalizumab) were reviewed. Individuals unresponsive to the standard doses of the second generation H1-antihistamines were categorized as difficult-to-treat cases. The length of disease duration after treatment was recorded and remission rate at 12 and 18 months were calculated. The duration from the onset of CSU with the presence of thyroid autoantibody to the development of AITD was evaluated. A review of laboratory tests related to urticaria were conducted (i.e. Complete blood count, erythrocyte sedimentation rate (ESR), antinuclear antibody (ANA), anti-TPO, anti-Tg, urine analysis (UA), and stool exam). ANA was performed by indirect immunofluorescent technique (EUROPattern ${ }^{\circledR}$, Euroimmun AG, Luebeck, Germany), a positive test was considered by titer $>1: 80$. Anti-TPO and anti-TG were performed by electro-chemiluminescence immunoassay (Elecsys ${ }^{\circledR}$, Roche Diagnostics GmbH, Mannheim, Germany). Results were positive if anti-TPO titer $>34 \mathrm{IU} / \mathrm{mL}$ or anti-TG $>115$ $\mathrm{IU} / \mathrm{mL}$. Patients were categorized as having TA if at least one anti-thyroid antibody was positive. Those with TA were further evaluated for thyroid function test (TFT). TFT (Abbott Diagnostics, Lake Forest, IL, USA), included thyroid stimulating hormone (TSH, reference range, 0.35$4.94 \mathrm{uIU} / \mathrm{mL}$ ), free triiodothyronine (FT3, reference range, 1.71-3.71 pg/mL), and free thyroxine (FT4, reference range, 0.7-1.48 ng/dL). AITDs were diagnosed by endocrinologists. Hashimoto's thyroiditis was diagnosed based on the demonstration of circulating thyroid antibodies and diffuse thyroid enlargement or reduced echogenicity on thyroid ultrasonography. The diagnosis of Graves' disease relies on persistent hyperthyroidism with positive thyroid antibody and/or increase vascularization on thyroid sonogram. The diagnosis of subclinical thyroid diseases was made when serum free $\mathrm{T} 4$ and free $\mathrm{T} 3$ levels remain within their respective reference ranges with the presence of abnormal TSH levels.

Regarding skin testing, ASST and APST were utilized as an in-vivo test to diagnose chronic autoimmune urticaria (CAU). Antihistamines were withheld 7 days prior to testing. Ten milliliters of venous blood were drawn to prepare the autologous serum and plasma. To perform the skin testing, $0.05 \mathrm{ml}$ of the autologous serum and plasma were injected intradermally into the volar side of each forearm. A negative control was done by using the same technique with $0.05 \mathrm{ml}$ of normal saline (NSS). Skin test reading was performed 30 minutes after the injections. ASST and APST were considered positive with induction of wheal diameter exceeded that of NSS by $1.5 \mathrm{~mm}$.

2.4. Statistical Analysis. Statistical analyses were conducted by STATA statistical software version 13 (Stata Corp LP, College station, TX, USA). To test for associations, the statistical methods such as Pearson's Chi-squared test and Fisher's exact test were used for categorical variables, while Student's t-test and Wilcoxon rank-sum test were operated for continuous variables with normal and non-normal distribution, respectively. Statistical significance was considered when $\mathrm{p}$-value $<0.05$. 


\section{Results}

Medical records of 1,096 patients diagnosed with urticaria were retrospectively reviewed, 463 patients were excluded due to the diagnosis of acute urticaria, urticarial vasculitis, inducible urticaria. Six-hundred sixty patients satisfied the diagnostic criteria for CSU (60.2\%), while 360 patients were excluded due to lack of one or both thyroid antibody results, giving a total of 300 study participants fulfilling the study inclusion criteria.

3.1. Patient Demographics. The majority of patients were female $(84.7 \%)$. Female gender was associated with TA, elevated anti-TPO level, and elevated anti-Tg level with statistical significance $(p=0.003, p=0.010$, and $p=0.024$, respectively). The mean age of CSU onset was $41.3 \pm 14.9$ years. Positive TA was significantly associated with CSU onset after 35 years of age compared to earlier age onset $(75 \%$ vs $57.6 \%, p=0.008)$. Fifty-four patients suffered from CSU with the presence of TA at latter onset. Of which $22.2 \%$ (12 patients) had AITDs consisting of Graves' disease 11.1\% (6 patients), Hashimoto's thyroid disease $5.6 \%$ (3 patients), subclinical hypothyroidism $3.7 \%$ (2 patients), and subclinical hyperthyroidism $1.9 \%$ (1 patient). The association between the onset of CSU and positive anti-Tg followed a similar direction $(78.2 \%$ vs. $58.1 \%$, respectively, $p=0.006)$. The same trend was shown for positive anti-TPO but without statistical significance $(69.8 \%$ vs. $60.1 \%, p=0.187)$. The disease duration ranged from 1.5-360 months (median 4 months). For atopic diathesis, allergic rhinitis was the most frequently reported $(54.9 \%)$. The occurrences of patients with history of nonsteroidal anti-inflammatory drug hypersensitivity, atopy, and dermographism, although not statistically significant, were higher in patients with TA. The parameters for CSU severity including frequency of attacks, wheal size, number of wheals, severity of itch, impairment of work, and disturbance of sleep were reported in percentage in Table 1.

In contrast to TA positivity, higher percent of CAU patients developed CSU before or at the age of 35 years compared to non-CAU (62.4\% vs. $37.5 \%, p=0.027)$. With statistical difference, approximately $64 \%$ of ASST-positive patients had history of atopy in comparison to $36.4 \%$ of ASSTnegative patients $(p=0.032)$. The presence of angioedema was more commonly noted in patients with CAU, ASST and APST positivity compared to those without CAU, or with negative ASST and APST $(p=0.058, p=0.183$, and $p=0.085$, respectively), shown in Table 2 .

3.2. Laboratory Analyses. Details of related laboratory findings are shown in Table 1. Anti-TPO and anti-Tg revealed positive for $17.7 \%$ and $18.3 \%$, respectively. Twenty-four percent had either anti-TPO or anti-Tg positivity while both tests were positive in 36 patients. (Table 2, Figures 1 and 2). The frequency of high-titer-ANA-positive patients was significantly correlated with higher frequency of TA, positive anti-TPO, and positive anti-Tg $(p=0.015, p=0.002$, and $p=0.046$, respectively). The frequency of patients showing elevated ESR was significantly higher in patients with TA and
anti-TPO positivity than those without $(p=0.035, p=0.024$, respectively).

Autologous skin testing was carried out in $125 \mathrm{CSU}$ patients, $101(80.8 \%)$ including 88 females $(87.1 \%)$ and 13 males $(12.9 \%)$ has positive results (Table 2, Figure 1). Both tests were positive in 79 patients (Figure 3). ASST and APST were positive in 91(72.8\%) and 88 (70.4\%) patients, respectively (Figures 1 and 3).The median time to perform the skin tests was 13 months (range 1.5-365 months) after CSU onset. There was no statistically significant association between TA and CAU ( $p=0.316)$ (Tables 1 and 2).

3.3. Thyroid Autoimmunity in Patients with CSU. Of 300 patients, high anti-TPO and anti-Tg were detected in 53 and 55 patients, respectively, and both thyroid autoantibodies were elevated in $12 \%$ (Figure 2). Seventy-two (24\%) patients with positivity to any thyroid autoantibodie(s) were diagnosed with TA and were subsequently evaluated for TFT. At the median time of 5 months from the CSU onset, AITDs were established in 10 patients (patient number 8, 10-12, and 14-18). By periodically repeating TFT, two more patients (patient number 9 and 13) were diagnosed with AITDs at the median time of 21 months from CSU onset. AITDs which corresponded to $15.3 \%$ (18/72; excluding patient number 3 due to negative thyroid autoantibodies) of patients showed positivity to either anti-TPO or anti-Tg. Seven patients suffered from AITDs prior to the onset of CSU (patient number 1-7). Information of CSU patients with AITDs is demonstrated in Table 3. All patients with AITDs were female. Graves' disease was the most common, followed by Hashimoto's disease. Other diagnoses included subclinical hypothyroidism, subclinical hyperthyroidism, and primary hypothyroidism.

\subsection{Thyroid Autoantibodies and Autoimmunity in CSU as a Predictor of CSU Prognosis}

3.4.1. Disease Severity. Generally, previously mentioned clinical parameters (see Methods) for CSU severity were not statistically different between patients with TA and the presence of anti-TPO and anti-Tg compared to those without.

As shown in Table 2, urticarial attacks > 4 days/week was more frequently reported in ASST- and APST-positive patients compared to ASST- and APST-negative patients ( $84.6 \%$ vs. $61.3 \%, p=0.011$, and $85.3 \%$ vs. $61.8 \%, p=0.006$ ). CAU patients had greater percentage of attacks by CSU $>4$ days/week than non-CAU patients but the statistical significance was not reached $(p=0.086)$. Likewise, $73.9 \%$ of patients with CAU experienced the number of wheals $>7$ lesions/day compared to $42.9 \%$ of those without ( $p=0.051$ ). In addition, the mean wheal diameter induced by autologous plasma was significantly associated with number of wheals $>$ 7 lesions/day (9.4 mm vs. $7.9 \mathrm{~mm}, p=0.037$ ). Other clinical and laboratory indicators for severity including wheal size, severity of itch and disturbance of sleep were not significantly correlated with either the presence of CAU, positive ASST, or positive APST. 


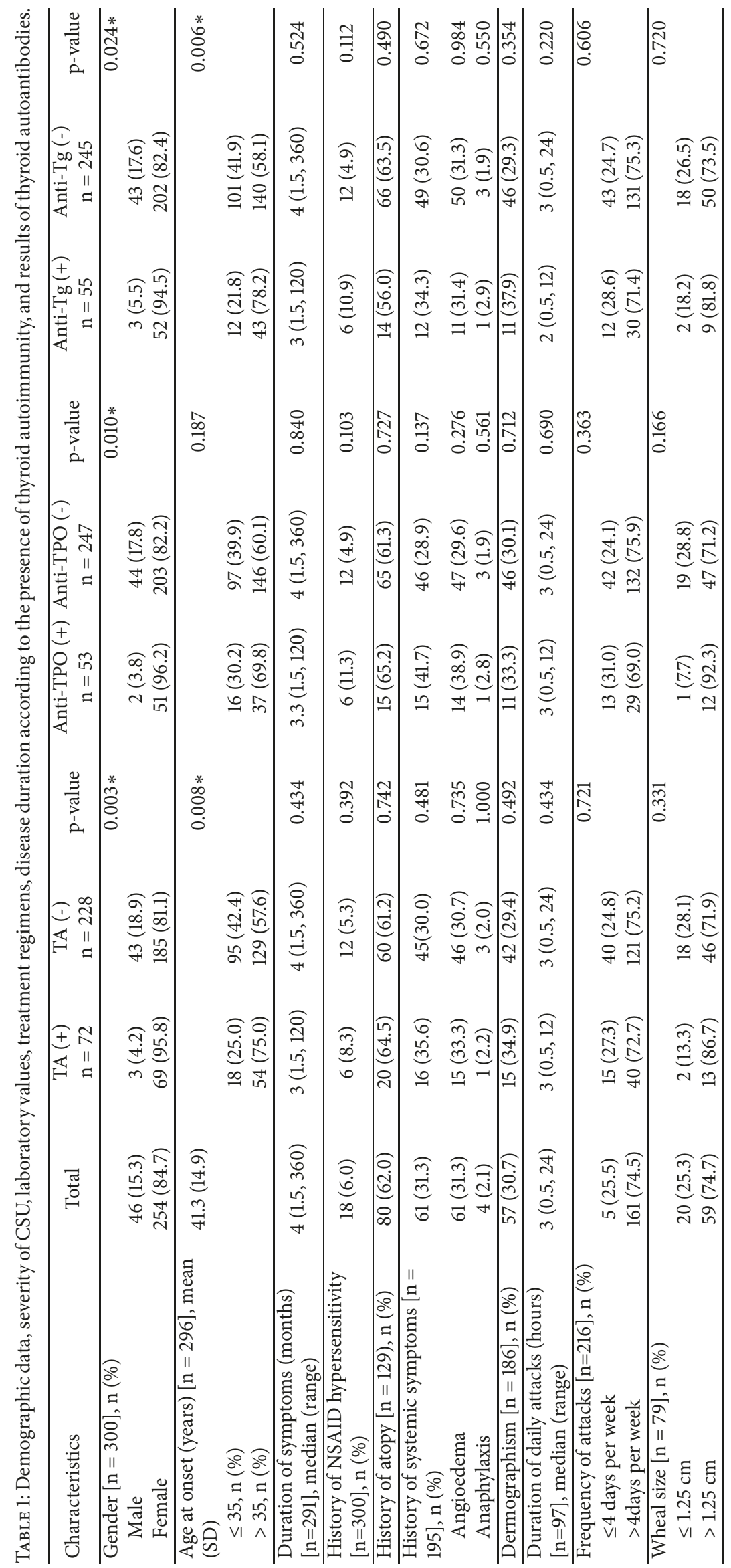




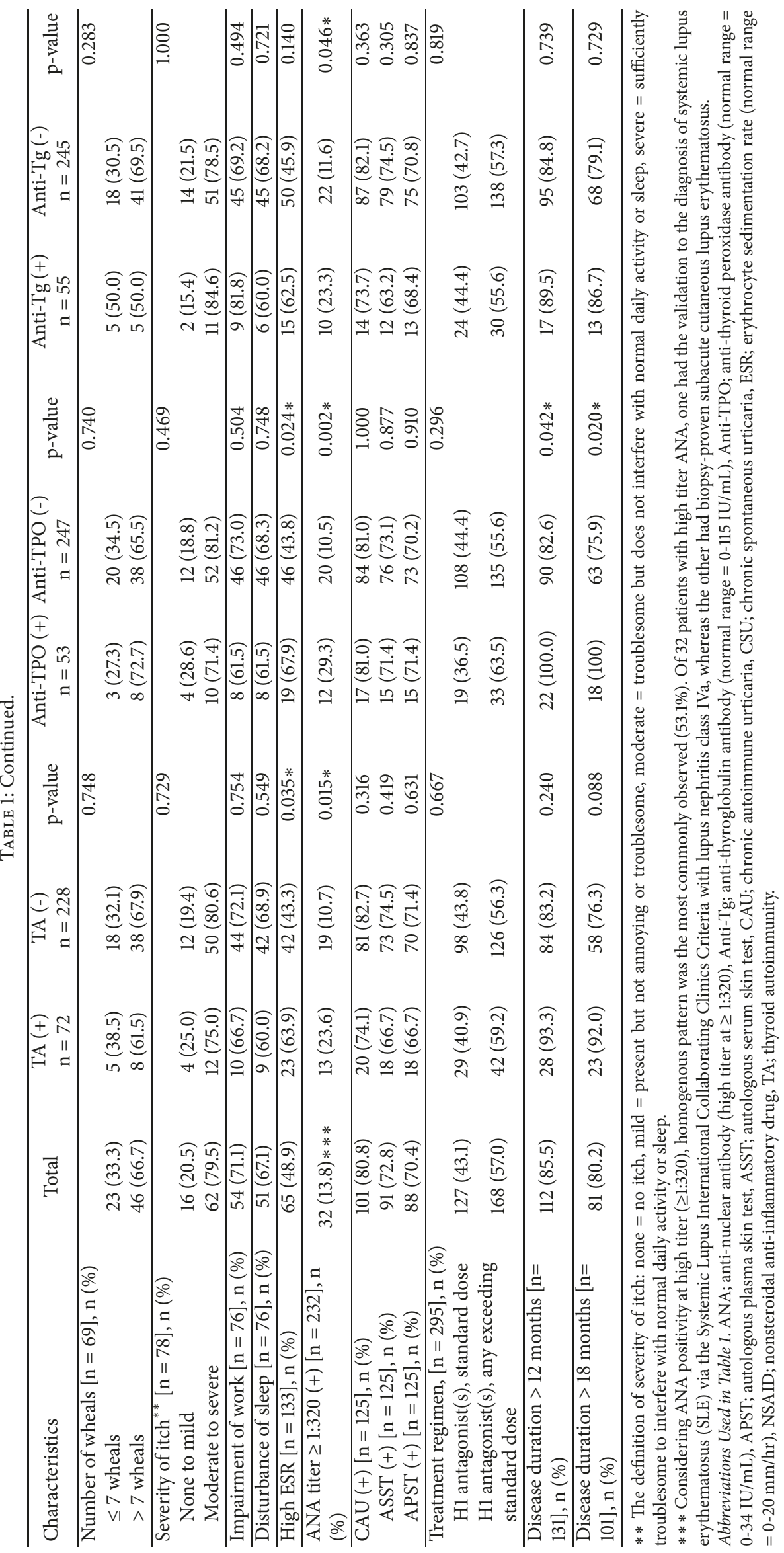




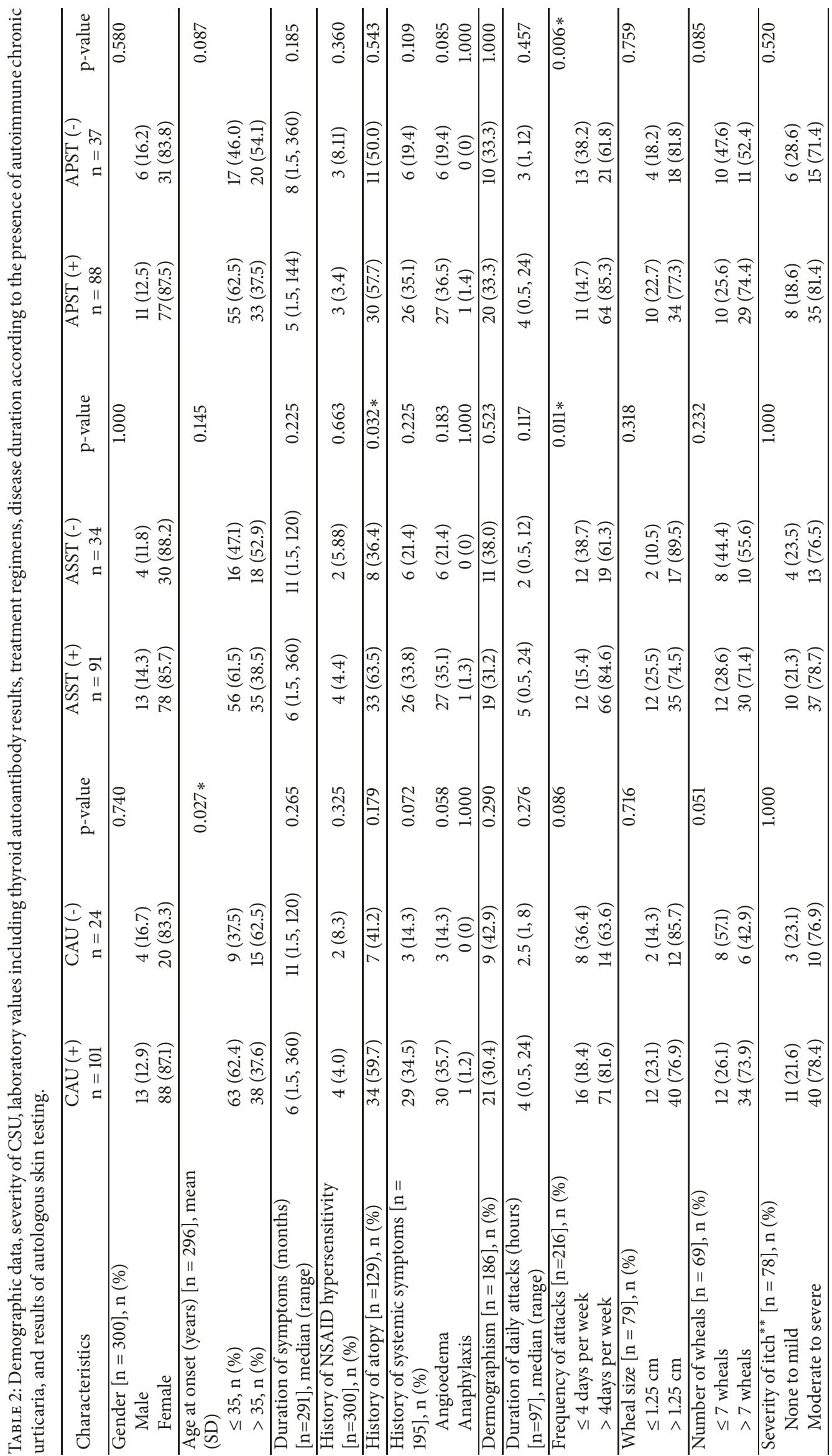




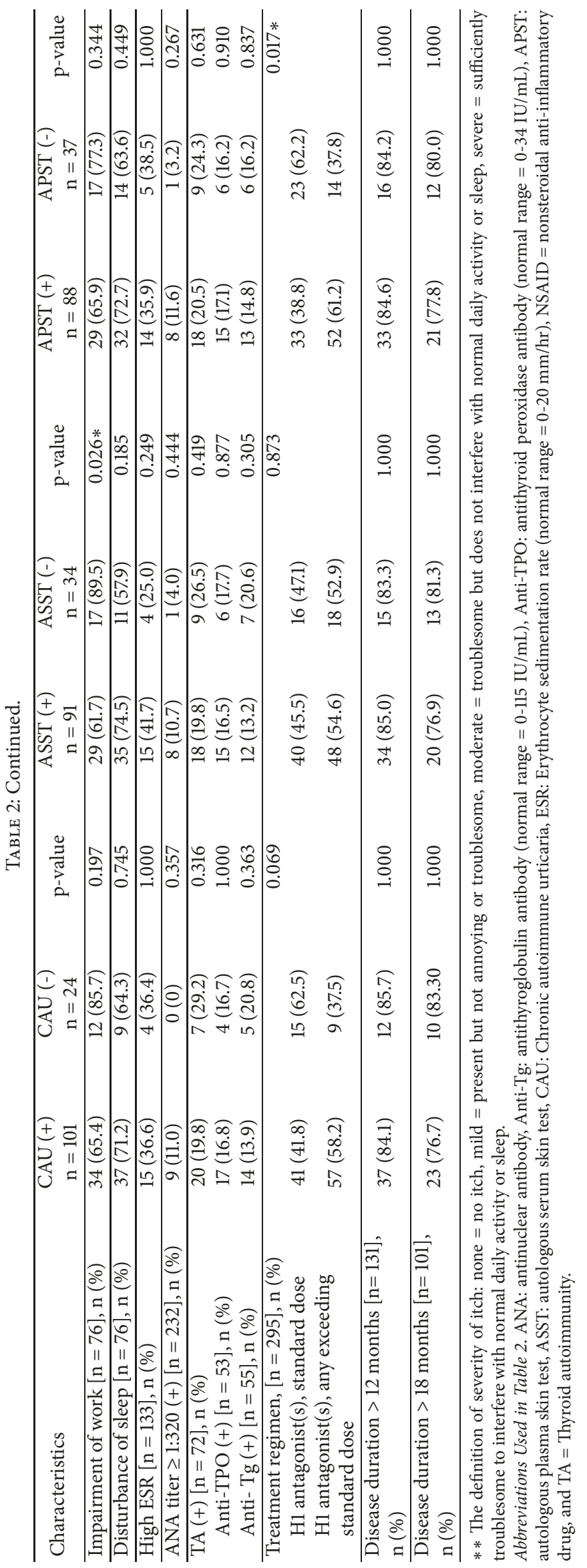




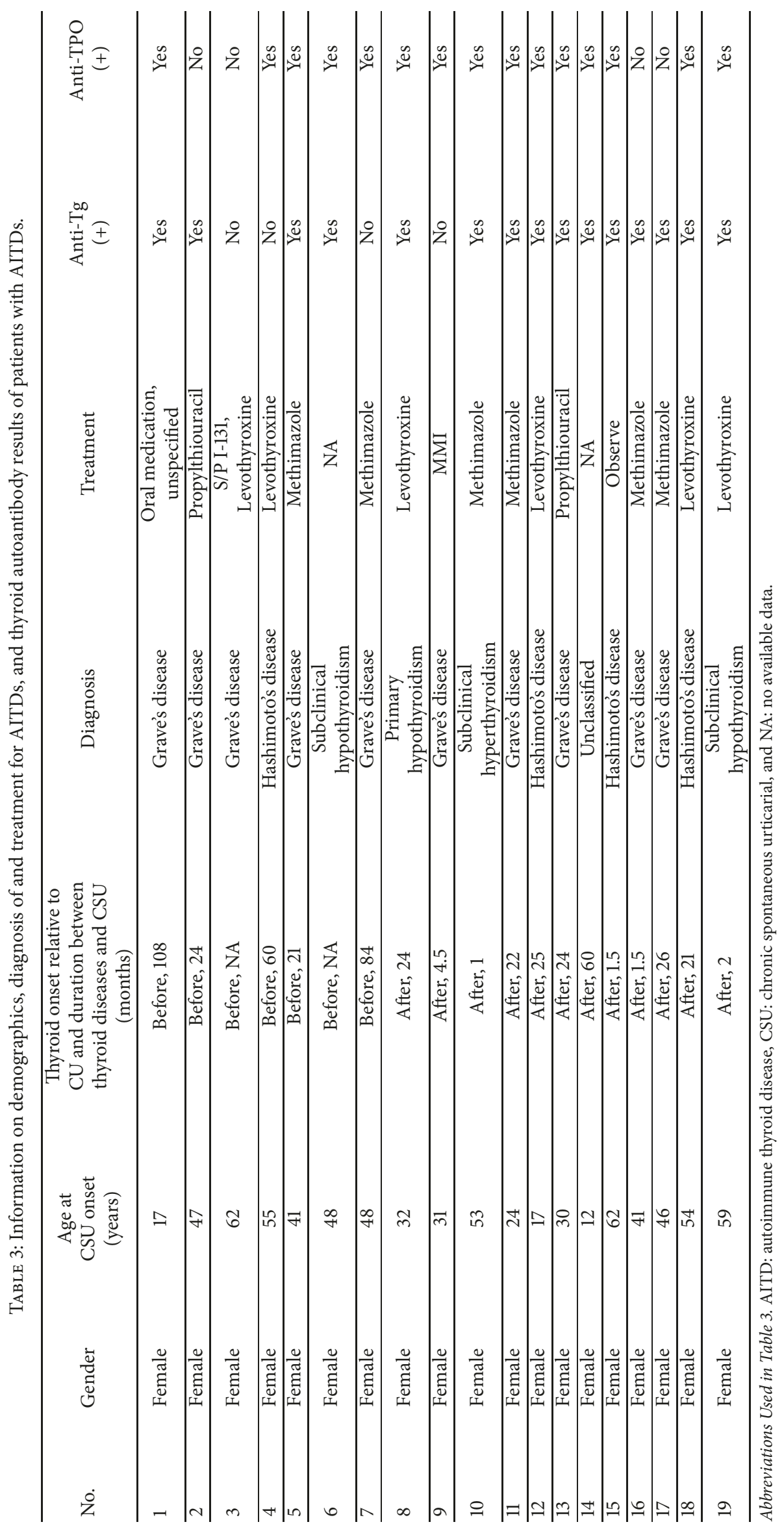




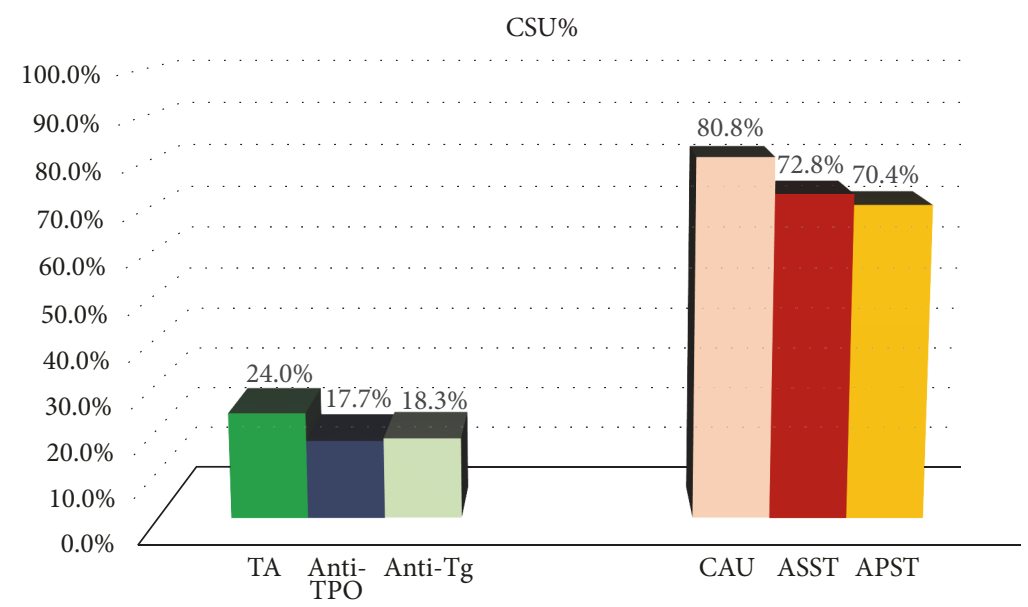

FIGURE 1: Percentages of chronic spontaneous urticaria patients who had thyroid autoimmunity (TA), positive anti-TPO (antithyroid peroxidase), positive anti-Tg (anti-thyroglobulin), chronic autoimmune urticaria (CAU), positive autologous serum skin test (ASST), and positive autologous plasma skin test (APST).

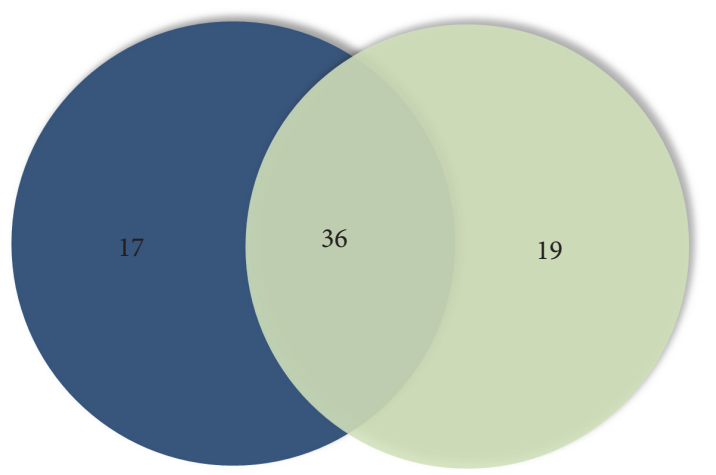

Positive anti-TPO

Positive anti-Tg

Positive both anti-Tg and anti-TPO

FIGURE 2: Number of CSU patients with positive antithyroid peroxidase (anti-TPO) and antithyroglobulin (anti-Tg).

3.4.2. Therapeutic Response. Second-generation H1-antihistamines exceeding standard dosages either given singly or in combination with other $\mathrm{Hl}$-antihistamine(s) were administered in over half of the patients $(57.0 \%)$ while the rest were controlled with standard-dose-H1-antihistamine (Table 1). Cyclosporin A, omalizumab, monteleukast, and $\mathrm{H} 2$-receptor antagonist were prescribed in 2, 1, 3, and 17 patients, respectively. The presence of TA, anti-TPO, or anti-Tg did not significantly influence therapeutic response ( $p=0.667, p=0.296$, and $p=0.819$, respectively).

In terms of CAU, patients with positive APST had higher frequency of commencing second generation H1antihistamine(s) exceeding standard dosages compared to negative APST ( $61.2 \%$ vs. $37.8 \%, p=0.017)$. The association of CAU and difficult-to-treat cases showed similar pattern but did not reach statistical significance $(p=0.069)$, however,

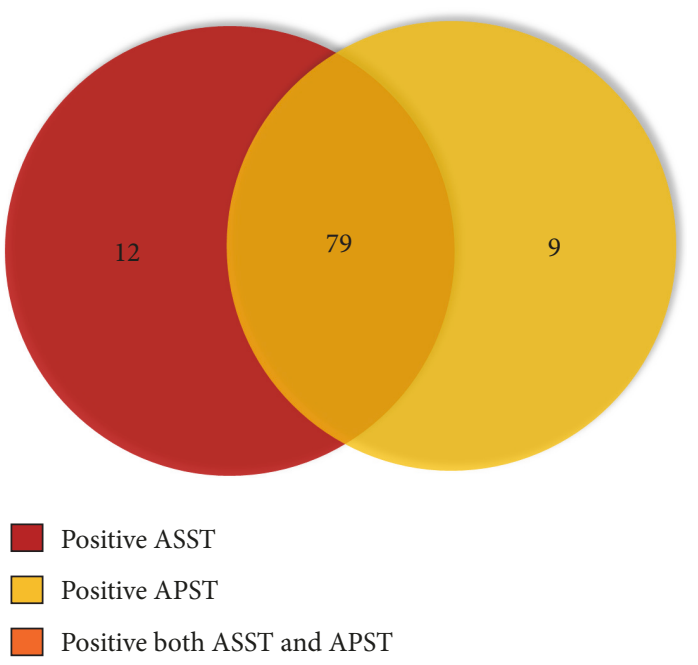

FIGURE 3: Number of CSU patients with positive autologous serum skin test (ASST) and autologous plasma skin test (APST).

ASST did not demonstrate differences in therapeutic regimens $(p=0.873)$ (Table 2).

3.4.3. Time to Remission. As shown in Table $1,85.5 \%$ and $80.2 \%$ suffered from persistent CSU of more than 12 and 18 months, respectively. Patients with TA had active disease longer than 12 and 18 months after therapy compared to those without ( $93.3 \%$ vs. $83.2 \%$ and $92.0 \%$ and $76.3 \%$, respectively) though the statistical difference was not reached $(p=0.240$ and $p=0.088$, respectively). A similar pattern was observed in anti-Tg-positive and anti-Tg-negative group. However, antiTPO-positive patients were significantly more prevalent in attaining persistent disease longer than 12 and 18 months (100\% vs. $82.6 \%, \mathrm{p}=0.042$ and $100 \%$ vs. $75.9 \%, p=0.020$, respectively). 
The percentages of patients with active CSU at 12 or 18 months, in CAU or non-CAU group, in ASST-positive or ASST-negative group, or in APST-positive or APST-negative group were not significantly different (Table 2).

\section{Discussion}

The clinical spectrum and outcome of patients with urticaria is variable, hence, multiple factors may be involved in the clinical and prognostic polymorphism. Early predictions on CSU as to whom would be expected to have severe, difficult-to-treat and/or long-lasting disease is desirable for proper patient education and appropriate management plans, therefore, our study is among the few to evaluate these factors in association to TA and autoimmunity in CSU.

Of 1,096 patients, $60.2 \%$ fulfilled the criteria for the diagnosis of CSU. The prevalence corresponds to the previous reports of $56-93 \%$. The frequency of TA was $24.0 \%(17.7 \%$ for anti-TPO and $18.3 \%$ for anti-Tg) and $6.3 \%$ had AITDs (Figure 1). Literature reports on the prevalence of TA ranged from $4.3-57 \%$, among this $5-10 \%$ have clinically apparent thyroid disease $[10,11]$. In a national survey of normal Thai patients, anti-TPO and anti-Tg were positive in $8.9 \%$ and $12.3 \%$, respectively [11]. Our study confirms higher prevalence of TA in the CSU population. We also highlight that AITDs could manifest as early as 9 years prior to or up to 5 years following CSU onset (Table 3). More importantly, many patients were diagnosed by subsequent TFT evaluation. We emphasize that periodic evaluation of TFT is crucial in CSU patients showing positive thyroid autoantibodies. The definite mechanism behind the association between TA and urticaria remains to be determined. However, postulated hypothesis for autoimmune CSU involves two mechanisms: type I and II autoimmunity [12]. In type I autoimmune CSU, IgE autoantibodies bind to high affinity mast cell receptor. Anti-TPO is one of the most common CSUassociated autoallergen identified [13]. In type II autoimmune CSU, particularly autoimmunity type IIb, IgG autoantibody is responsible for mast cell degranulation via activation of high-affinity IgE receptor. The definitive diagnosis of this type relies on positive autologous skin test and/or BAT and histamine release test [13]. Evidence have shown that there is a strong link between elevated levels of IgG antithyroid antibodies and CSU [14]. Therefore, autoimmune CSU in our subpopulation could possibly be classified as autoimmunity type IIb. Genetic and environmental factors could also be attributed. Another possible pathogenesis is that antithyroid drugs, i.e., methimazole, carbimazole, or propylthiouracil, may cause itching and urticaria as seen in several patients in the present study commencing these medication prior to the onset of CSU (Table 3) [15]. Moreover, exposure to specific circulating antigen particularly as a result of autoimmune thyroid damage, anti-TPO IgE is produced and may potentially induce urticarial symptoms, mast cell sensitization and degranulation [16]. Nevertheless, to date, there is insufficient evidence to prove that thyroid autoantibodies are pathogenic for CSU and studies have failed to demonstrate cross-reactivity between antithyroid antibody and other autoantibodies in CSU. In addition, antithyroid antibodies alone are not capable of inducing mast cell activation [17].

In agreement with other reports, TA is more prevalent in females (female: male 5.5:1) [18-20]. The proposed mechanisms for female preponderance may involve the underlying state of inflammation driven by adipokines, especially leptin, TNF- $\alpha$, and IL-6, and several receptors including Toll-like receptors on thyrocytes. Because leptin levels are higher in females, the function of thyrocytes in innate immunity fails to act properly against triggers such as viruses, bacteria, and stress. This then contributes to the initiation step to break tolerance to thyroid self-antigens [19]. The mean age onset of our patients was $41.3 \pm 14.9$ years. This was similar to that demonstrated in the literature $[18,20]$. To the best of our knowledge, we are the first to report the statistically significant association between the presence of TA and the age of CSU onset older than 35 years. Regarding ANA testing, the percentage of patients showing positive ANA titer $>1: 320$ was slightly lower than that of a recent report (17.5\%) [20]. This difference can be partially justified by different groups of population and ethnicity. Of note, our study highlights the significant correlation between TA and positive hightiter ANA among CSU patients. This supports earlier studies in CSU showing the association between ANA and TA [21, 22]. It is believed that the presence of non-organ-specific autoantibodies such as ANA, may demonstrate a polyclonally accelerated production of autoantibodies by immune cells and also thyrocytes [22]. However, the presence of ANA titer $\geq 1: 320$ can be found in $1.4 \%$ of the healthy population [23]. Moreover, of 32 patients with positive high-titer ANA, only 2 were diagnosed with lupus erythematosus. Therefore, the presence of positive ANA may not necessarily be pathologic and the clinical significance of ANA positivity remains to be determined.

Our findings suggested that early age onset of before 35 years may predict autoimmune basis of CSU. This is compatible with the earlier study reporting that patients with CAU were relatively younger than non-CAU patients [24]. Sharing IgE-mediated mechanism, CSU is believed to be associated with allergic dysentery. We found a strong association between atopy and ASST positivity. Reports in the literature are mostly but not always consistent with this [24, 25]. Regarding ANA positivity, although strong association was lacking, it was more prevalent in CAU patients. Larger number of participants may yield more apparent results.

Our study demonstrated a relatively higher frequency of CAU patients (80.8\%) and also ASST (72.8\%) and APST positivity $(70.4 \%)$ compared to previous literature [25-27]. However, similar to our findings, reports of considerably high positivity for ASST (66\%) and APST (86\%) have been demonstrated $[28,29]$. An explanation for these discrepancies could be from differences in the study population, patient selection, and criteria adopted to score the test [30]. Unlike some reports, we did not find an association between positive autologous skin testing and the presence of antithyroid antibodies [18, 31]. However, our finding is consistent with those of Kocatürk, Yadav, and Alpay et al. [32-34]. Therefore, the association between CAU and TA remains controversial and 
requires further validation. The presence of TA or positive anti-TPO could serve as a predictor for elevated ESR but not for other clinical parameters. We believe that it is appropriate to monitor ESR in patients with CSU which represents a chronic inflammatory condition. Our study showed that at the end of 12 months, symptoms of CSU persisted in $85.5 \%$ of participants and $80.2 \%$ existed after 18 months. These figures are relatively high compared to previous reports [27, $35,36]$. This could be due to the high referral rate and more severe and persistent CSU patients sent to our specialized outpatient clinic. Of the thyroid antibodies, anti-TPO alone played a significant role in predicting persistent disease of CSU. An explanation to longer CSU duration related to thyroid antibody remains unclear. A possible mechanism is that long-lasting CSU may result from prolonged T-cell stimulation followed by extended polyclonal activation and the production of various inflammatory mediators. This could possibly induce the production of other autoantibodies such as anti-TPO. Toubi et al. also found that thyroid antibody is associated with CSU disease duration [27]. In our study, only anti-TPO, not anti-Tg, predicted longer disease duration. Anti-TPO is indeed more sensitive and specific than antiTg for TA [37]. Moreover, anti-TPO also has superiority over anti-Tg for detecting AITDs such as Graves' disease and Hashimoto thyroiditis [38]. In addition, autoantibodies against the complement controller domain of TPO can activate complement through the classical pathway and raise $\mathrm{C} 4 \mathrm{a}$ levels at baseline normalized in remission after treatment with levothyroxine. However, the potential role of anti-TPO in the pathogenesis of CSU remains to be determined [39].

The current study reinforced previous findings that ASST and/or APST could serve as a predictor for CSU severity including higher wheal number and more frequent attacks $[26,40,41]$. Interestingly, not only is the skin testing associated with CSU activity, but the wheal diameter could predict disease severity as well. Although, autologous skin testing did not show benefit in predicting disease duration, our results proved that APST can be used as a potential marker to predict difficult-to-treat cases requiring antihistamines exceeding standard dosing. Our results are in line with that of Staubach et al. proving that ASST positive patients were likely to use more antihistaminic medication than ASST negative patients [42]. Viswanathan et al. also demonstrated that commercially available basophil histamine release assay had strong association to refractoriness of antihistamines [20]. There is controversy regarding the advantage of APST and ASST over the other. Few studies have shown that APST gave higher percentage of positive result than ASST as coagulation factors in plasma might have a pivotal role in pathogenesis and severity of CSU [29, 43], whereas others have shown indifferences between them $[44,45]$. Based on our finding, we believe that APST could be a parameter to predict therapeutic response in CSU; however, more research is needed to validate this finding

The limitation of this study includes its retrospective nature. Subjective evaluation of medical record was performed rather than using a validated instrument for disease severity such as the urticaria activity score [46]. Autoimmune CSU was confirmed by positive ASST and APST results, while
BAT and histamine release test was not applied. Moreover, this study lacked the standard protocol for management. Step-wise algorithm for the treatment of CSU was not given to all patients; e.g., some patients were utilizing $2 \mathrm{x}$ daily antihistamine or more than one type of antihistamine, rather than the current recommended $4 \mathrm{x}$ dosing [46]. Finally, all patients were collected from a single center at a tertiary institution which may represent a more severe subgroup of CSU due to referral bias. However, the large sample size and the unified protocol for evaluation for all parameters conduced in this study should give more information regarding TA and autoimmunity in CSU linked to the disease severity and prognosis in CSU. Nevertheless, future prospective work is warranted to confirm our results.

In conclusion, we demonstrate that anti-TPO can potentially indicate longer CSU disease duration while autoimmunity in CSU can predict disease severity and therapeutic response. We recommend evaluating thyroid antibodies in patients with CSU particularly in females, patients $>35$ years of age. Moreover, in all CSU patients especially $<35$ years old, autologous skin testing is highly suggested.

\section{Data Availability}

The data used to support the finding of this study are available from the corresponding author upon request.

\section{Conflicts of Interest}

The authors have no conflicts of interest to declare.

\section{References}

[1] X.-F. Pan, J.-Q. Gu, and Z.-Y. Shan, “The prevalence of thyroid autoimmunity in patients with urticaria: a systematic review and meta-analysis," Endocrine Journal, vol. 48, no. 3, pp. 804810,2015

[2] M. Szyper-Kravitz, I. Marai, and Y. Shoenfeld, "Coexistence of thyroid autoimmunity with other autoimmune diseases: Friend or foe? Additional aspects on the mosaic of autoimmunity," Autoimmunity, vol. 38, no. 3, pp. 247-255, 2005.

[3] M. Maurer, K. Weller, C. Bindslev-Jensen et al., "Unmet clinical needs in chronic spontaneous urticaria. A GA ${ }^{2} \mathrm{LEN}$ task force report," Allergy, vol. 66, no. 3, pp. 317-330, 2011.

[4] T. Zuberbier, M. Balke, M. Worm, G. Edenharter, and M. Maurer, "Epidemiology of urticaria: a representative cross-sectional population survey," Clinical and Experimental Dermatology, vol. 35, no. 8, pp. 869-873, 2010.

[5] R. A. Sabroe, E. Fiebiger, D. M. Francis et al., "Classification of anti-Fce RI and anti-IgE autoantibodies in chronic idiopathic urticaria and correlation with disease severity," The Journal of Allergy and Clinical Immunology, vol. 110, no. 3, pp. 492-499, 2002.

[6] L. Curto-Barredo, J. Yelamos, R. Gimeno, S. Mojal, R. M. Pujol, and A. Giménez-Arnau, "Basophil Activation Test identifies the patients with Chronic Spontaneous Urticaria suffering the most active disease," Immunity, Inflammation and Disease, vol. 4, no. 4, pp. 441-445, 2016.

[7] R. A. Sabroe, P. T. Seed, D. M. Francis, R. M. Barr, A. Kobza Black, and M. W. Greaves, "Chronic idiopathic urticaria: 
comparison of the clinical features of patients with and without anti-FceRI or anti-IgE autoantibodies," Journal of the American Academy of Dermatology, vol. 40, no. 3, pp. 443-450, 1999.

[8] R. Confino-Cohen, G. Chodick, V. Shalev, M. Leshno, O. Kimhi, and A. Goldberg, "Chronic urticaria and autoimmunity: Associations found in a large population study," The Journal of Allergy and Clinical Immunology, vol. 129, no. 5, pp. 1307-1313, 2012.

[9] Y. S. Kim, K. Han, J. H. Lee et al., "Increased Risk of Chronic Spontaneous Urticaria in Patients With Autoimmune Thyroid Diseases: A Nationwide, Population-based Study," Allergy, Asthma \& Immunology Research, vol. 9, no. 4, p. 373, 2017.

[10] Y. Levy, N. Segal, N. Weintrob, and Y. L. Danon, "Chronic urticaria: Association with thyroid autoimmunity," Archives of Disease in Childhood, vol. 88, no. 6, pp. 517-519, 2003.

[11] C. Sriphrapradang, S. Pavarangkoon, W. Jongjaroenprasert, L.O. Chailurkit, B. Ongphiphadhanakul, and W. Aekplakorn, "Reference ranges of serum TSH, FT4 and thyroid autoantibodies in the Thai population: The national health examination survey," Clinical Endocrinology, vol. 80, no. 5, pp. 751-756, 2014.

[12] P. Kolkhir, M. K. Church, K. Weller, M. Metz, O. Schmetzer, and M. Maurer, "Autoimmune chronic spontaneous urticaria: What we know and what we do not know," The Journal of Allergy and Clinical Immunology, vol. 139, no. 6, pp. 1772-1781.el, 2017.

[13] M. Maurer, T. Zuberbier, F. Siebenhaar, and K. Krause, "Chronic urticaria - What does the new guideline tell us?" JDDG: Journal der Deutschen Dermatologischen Gesellschaft, vol. 16, no. 5, pp. 584-593, 2018.

[14] P. Kolkhir, M. Metz, S. Altrichter, and M. Maurer, "Comorbidity of chronic spontaneous urticaria and autoimmune thyroid diseases: A systematic review," Allergy: European Journal of Allergy and Clinical Immunology, vol. 72, no. 10, pp. 1440-1460, 2017.

[15] M. Bagnasco, P. L. Minciullo, M. Schiavo, G. Saraceno, S. Gangemi, and S. Benvenga, "Urticaria and thyroid autoimmunity," Thyroid, vol. 21, no. 4, pp. 401-410, 2011.

[16] M. Rottem, "Chronic urticaria and autoimmune thyroid disease: is there a link?" Autoimmunity Reviews, vol. 2, no. 2, pp. 69-72, 2003.

[17] J. D. Mozena, A. Tĩana, J. Negri, J. W. Steinke, and L. Borish, "Lack of a role for cross-reacting anti-thyroid antibodies in chronic idiopathic urticaria," Journal of Investigative Dermatology, vol. 130, no. 7, pp. 1860-1865, 2010.

[18] B. F. O’Donnell, D. M. Francis, G. T. Swana, P. T. Seed, A. K. Black, and M. W. Greaves, "Thyroid autoimmunity in chronic urticaria," British Journal of Dermatology, vol. 153, no. 2, pp. 331335, 2005.

[19] S. J. Merrill and Y. Mu, "Thyroid autoimmunity as a window to autoimmunity: An explanation for sex differences in the prevalence of thyroid autoimmunity," Journal of Theoretical Biology, vol. 375, pp. 95-100, 2015.

[20] R. K. Viswanathan, M. J. Biagtan, and S. K. Mathur, "The role of autoimmune testing in chronic idiopathic urticaria," Annals of Allergy, Asthma \& Immunology, vol. 108, no. 5, pp. 337-341, 2012.

[21] M. G. Tektonidou, M. Anapliotou, P. Vlachoyiannopoulos, and H. M. Moutsopoulos, "Presence of systemic autoimmune disorders in patients with autoimmune thyroid diseases," Annals of the Rheumatic Diseases, vol. 63, no. 9, pp. 1159-1161, 2004.
[22] I. Lazúrová, K. Benhatchi, J. Rovenský et al., "Autoimmune thyroid disease and autoimmune rheumatic disorders: A twosided analysis," Annals of the New York Academy of Sciences, vol. 1173, pp. 211-216, 2009.

[23] S. A. S. V. Fernandez, A. Z. O. C. Lobo, Z. N. A. P. D. Oliveira, L. M. A. I. Fukumori, A. M. A. P rigo, and E. A. Rivitti, "Prevalence of antinuclear autoantibodies in the serum of normal blood dornors," Revista do Hospital das Clínicas, vol. 58, no. 6, pp. 315319, 2003.

[24] T. Boonpiyathad and A. Sangasapaviliya, "Autologous serum and plasma skin test to predict 2-year outcome in chronic spontaneous urticaria," Asia Pacific Allergy, vol. 6, no. 4, pp. 226-235, 2016.

[25] K. Kulthanan, S. Jiamton, T. Gorvanich, and S. Pinkaew, "Autologous serum skin test in chronic idiopathic urticaria: Prevalence, correlation and clinical implications," Asian Pacific Journal of Allergy and Immunology, vol. 24, no. 4, pp. 201-206, 2006.

[26] M. Metz, A. Giménez-Arnau, E. Borzova, C. E. Grattan, M. Magerl, and M. Maurer, "Frequency and clinical implications of skin autoreactivity to serum versus plasma in patients with chronic urticaria," The Journal of Allergy and Clinical Immunology, vol. 123, no. 3, pp. 705-706, 2009.

[27] E. Toubi, A. Kessel, N. Avshovich et al., "Clinical and laboratory parameters in predicting chronic urticaria duration: A prospective study of 139 patients," Allergy: European Journal of Allergy and Clinical Immunology, vol. 59, no. 8, pp. 869-873, 2004.

[28] V. Sajedi, M. Movahedi, A. Aghamohammadi et al., "Comparison between sensitivity of autologous skin serum test and autologous plasma skin test in patients with Chronic Idiopathic Urticaria for detection of antibody against IgE or IgE receptor (FcepsilonRIalpha," Iranian Journal of Allergy, Asthma and Immunology, vol. 10, pp. 111-117, 2011.

[29] R. Asero, A. Tedeschi, P. Riboldi, and M. Cugno, "Plasma of patients with chronic urticaria shows signs of thrombin generation, and its intradermal injection causes wheal-and-flare reactions much more frequently than autologous serum," The Journal of Allergy and Clinical Immunology, vol. 117, no. 5, pp. 1113-1117, 2006.

[30] R. Asero, M. Cugno, and A. Tedeschi, "Autologous plasma and serum skin test in chronic urticaria," British Journal of Dermatology, vol. 166, no. 6, pp. 1362-1363, 2012.

[31] S. Lunge, S. Pande, and M. Borkar, "Correlation of serum antithyroid microsomal antibody and autologous serum skin test in patients with chronic idiopathic urticaria," Indian Dermatology Online Journal (IDOJ), vol. 6, no. 4, p. 248, 2015.

[32] E. Kocatürk, M. Kavala, E. Kural, S. Sarigul, and I. Zindanci, "Autologous serum skin test vs autologous plasma skin test in patients with chronic urticaria: Evaluation of reproducibility, sensitivity and specificity and relationship with disease activity, quality of life and anti-thyroid antibodies," European Journal of Dermatology, vol. 21, no. 3, pp. 339-343, 2011.

[33] A. Alpay, N. Solak Tekin, I. Ö. Tekin, H. C. Altinyazar, R. Koca, and S. Çnar, "Autologous serum skin test versus autologous plasma skin test in patients with chronic spontaneous urticaria," Dermatology Research and Practice, vol. 2013, 2013.

[34] S. Yadav, A. J. Kanwar, D. Parsad, and R. W. Minz, "Chronic idiopathic urticaria and thyroid autoimmunity: Perplexing association," Indian Journal of Dermatology, vol. 58, no. 4, p. 325, 2013.

[35] M. M. A. Kozel, J. R. Mekkes, P. M. M. Bossuyt, and J. D. Bos, "Natural course of physical and chronic urticaria and 
angioedema in 220 patients," Journal of the American Academy of Dermatology, vol. 45, no. 3, pp. 387-391, 2001.

[36] K. Kulthanan, S. Jiamton, N. Thumpimukvatana, and S. Pinkaew, "Chronic idiopathic urticaria: Prevalence and clinical course," The Journal of Dermatology, vol. 34, no. 5, pp. 294-301, 2007.

[37] L. Verneuil, C. Leconte, J. J. Ballet et al., "Association between chronic urticaria and thyroid autoimmunity: A prospective study involving 99 patients," Dermatology, vol. 208, no. 2, pp. 98-103, 2004.

[38] E. Fröhlich and R. Wahl, "Thyroid Autoimmunity: Role of Antithyroid Antibodies in Thyroid and Extra-Thyroidal Diseases," Frontiers in Immunology, vol. 8, 2017.

[39] J. M. Stitt and S. C. Dreskin, "Urticaria and autoimmunity: Where are we now?" Current Allergy and Asthma Reports, vol. 13, no. 5, pp. 555-562, 2013.

[40] S. Alyasin, M. Hamidi, A. A. Karimi, A. Amiri, F. Ghaffarpasand, and M. J. Ehsaei, "Correlation between clinical findings and results of autologous serum skin test in patients with chronic idiopathic urticaria," Southern Medical Journal, vol. 104, no. 2, pp. 111-115, 2011.

[41] M. Abd El-Azim and S. Abd El-Azim, "Chronic autoimmune urticaria: Frequency and association with immunological markers," Journal of Investigational Allergology and Clinical Immunology, vol. 21, no. 7, pp. 546-550, 2011.

[42] P. Staubach, K. Onnen, A. Vonend et al., "Autologous whole blood injections to patients with chronic urticaria and a positive autologous serum skin test: A placebo-controlled trial," Dermatology, vol. 212, no. 2, pp. 150-159, 2006.

[43] S. Aktar, N. Akdeniz, H. U. Ozkol, O. Calka, and A. S. Karadag, "The relation of autologous serum and plasma skin test results with urticarial activity score, sex and age in patients with chronic urticaria," Postepy Dermatologii i Alergologii, vol. 32, no. 3, pp. 173-178, 2015.

[44] H. Yildiz, O. Karabudak, B. Doğan, and Y. Harmanyeri, "Evaluation of autologous plasma skin test in patients with chronic idiopathic urticaria," British Journal of Dermatology, vol. 165, no. 6, pp. 1205-1209, 2011.

[45] K. Thadanipon and P. Wattanakrai, "Comparison between autologous serum skin test and autologous plasma skin test in Thai chronic urticaria patients," Journal of the Medical Association of Thailand, vol. 100, no. 9, pp. 1014-1020, 2017.

[46] T. Zuberbier, W. Aberer, R. Asero et al., "The EAACI/GA(2) LEN/EDF/WAO Guideline for the definition, classification, diagnosis, and management of urticaria: the 2013 revision and update," Allergy, vol. 69, pp. 868-887, 2014. 


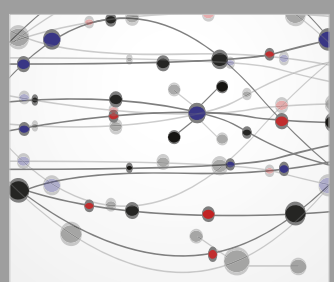

The Scientific World Journal
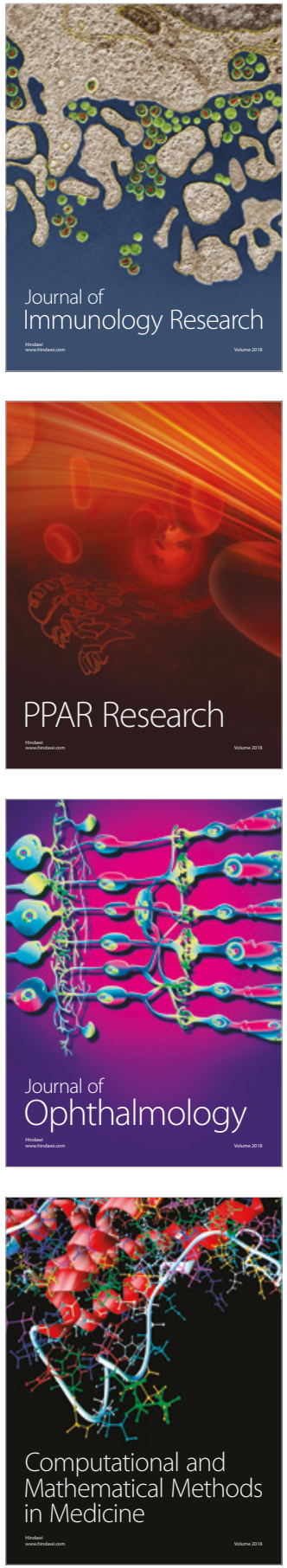

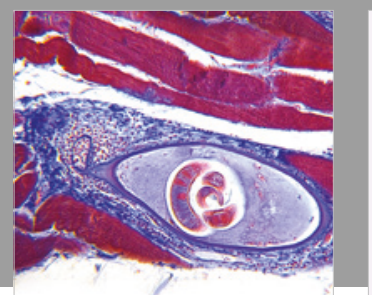

Gastroenterology Research and Practice

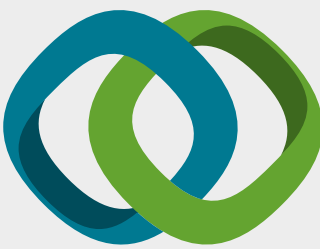

\section{Hindawi}

Submit your manuscripts at

www.hindawi.com
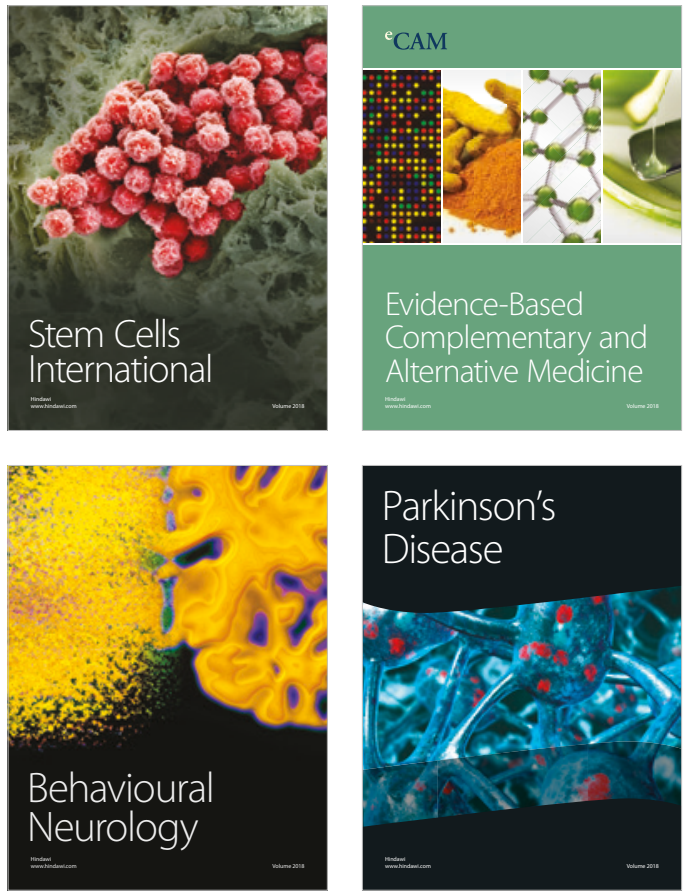

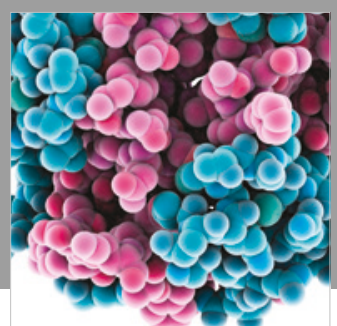

ournal of

Diabetes Research

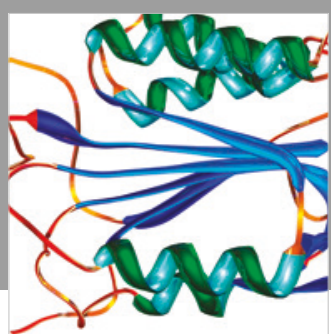

Disease Markers
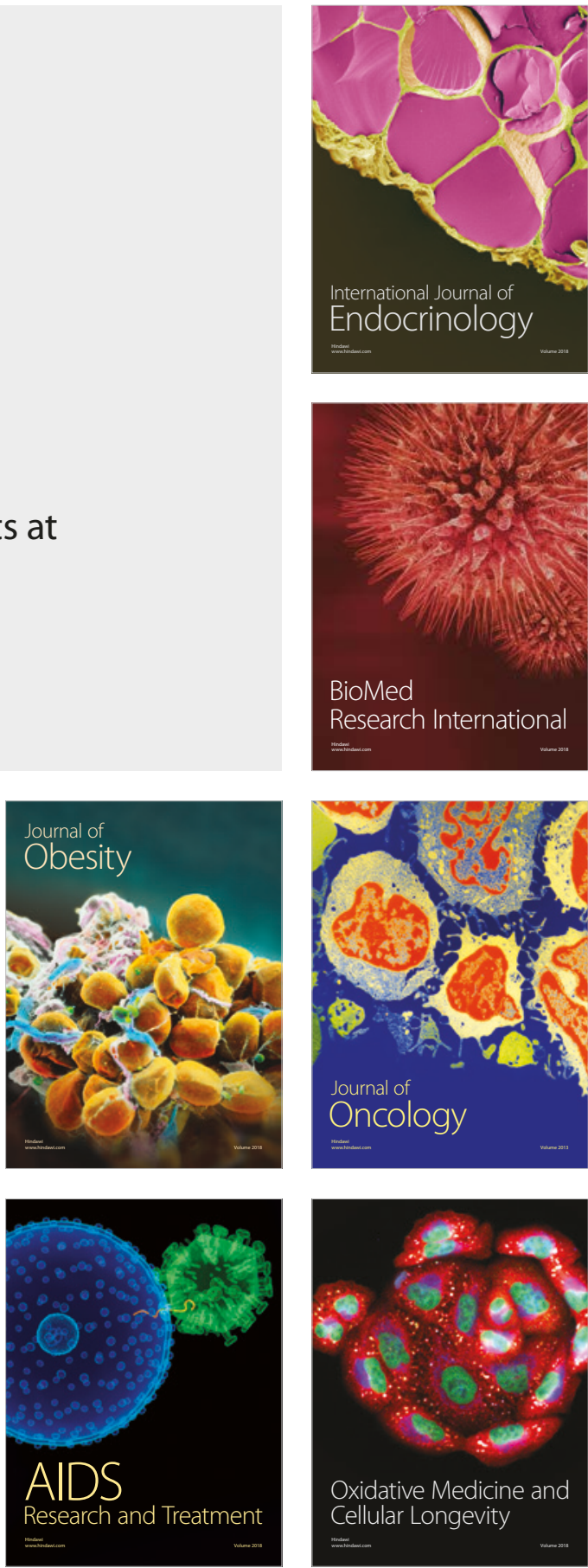